\title{
Deriving the Composite Simpson Rule by Using Bernstein Polynomials for Solving Volterra Integral Equations
}

\author{
Jenan Ahmad Al-A'asam* \\ Received 12, December, 2012 \\ Accepted 8, May, 2013
}

\begin{abstract}
:
In this paper we use Bernstein polynomials for deriving the modified Simpson's 3/8 , and the composite modified Simpson's 3/8 to solve one dimensional linear Volterra integral equations of the second kind, and we find that the solution computed by this procedure is very close to exact solution.
\end{abstract}

Key words:Integral equation, Bernstein polynomial, Simpson rule.

\section{Introduction:}

Integral equations are equations in which the unknown function appears under the sign of integral [1]. It is well known that integral equations arise in many branches of science, for example biological species [2],[3], sliding a bead along a wire [4], human population[4].Also integral equations have a relation with initial and boundary value problems[1],[3].

The theoretical methods for solving Volterra integral equations aresuccessiveapproximation,

successive substitution, Laplace transformation, Adomian decomposition and series solution methods. Many researchers study the numerical

solution[4],[5],[6],[7],[8],[9].

Block-by-block method is used for solving linear Volterra integral equations [10]. Quadrature

method is used for solving linear Volterra integral equations of the second kind [11],[12].

Volterra Integral Equations: [1],[3]
The general form of Volterra integral equation is

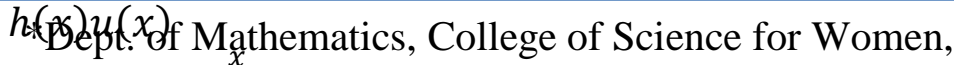

$$
\begin{aligned}
& =f(x)+\lambda \int_{a} R(x, y, u(y)) d y
\end{aligned}
$$

and this equation is said to be :

- Volterra integral equation of the first kind if $h(x)=0$.

- Volterra integral equation of the second kind if $h(\mathrm{x})=1$.

- Linear if $R(x, y, u(y))=$ $k(x, y) u(y)$, otherwise it is nonlinear.

- Homogeneous if $f(x)=$

0 , otherwise it is nonhomogeneous.

And,for more details see[1].

\section{Bernstein Polynomials [13]:}

Bernstein polynomials are defined by

$$
B_{i, n}(t)=\left(\begin{array}{l}
n \\
i
\end{array}\right) t^{i}(1-t)^{n-i}
$$

Where $\left(\begin{array}{c}n \\ i\end{array}\right)=\frac{n !}{i !(n-i) !}$

They are $n+1$ polynomials of degree n. For mathematical Convenience, weusually set $\quad B_{i, n}=0 \quad$ if $i<$ 0 or $i>n$.

For $\mathrm{n}=1$

$B_{0,1}(t)=1-t \quad$ and $\quad B_{1,1}(t)=t$

For $\mathrm{n}=2$ 


$$
\begin{aligned}
& B_{0,2}(t)=(1-t)^{2}, \quad B_{1,2}(t) \\
& =2 t(1 \\
& -t) \text { and } B_{2,2}(t)=t^{2}
\end{aligned}
$$

A recursive definition of Bernstein polynomials is given by

$$
\begin{aligned}
B_{i, n}(t)=(1-t) B_{i, n-1}(t) \\
+t B_{i-1, n-1}(t)
\end{aligned}
$$

These polynomials are non-negative over the interval $[0,1]$ and form a partition of unity

$$
\begin{aligned}
{\left[B_{0,1}(t)+B_{1,1}\right.} & (t) \\
& =1, B_{0,2}(t)+B_{1,2}(t) \\
& +B_{2,2}(t) \\
& =1 \text { and so on }]
\end{aligned}
$$

The Modified Simpson's 3/8 Rule:

By the Bernstein polynomials

$$
\sum_{k=0}^{n} f\left(\frac{k}{n}\right)\left(\begin{array}{l}
n \\
k
\end{array}\right) x^{k}(1-x)^{n-k}
$$

Where $f$ is a function, $k=0,1, \ldots, n$.

Then

$$
\begin{aligned}
& P(x)=f\left(\frac{0}{n}\right)\left(\begin{array}{l}
n \\
0
\end{array}\right) x^{0}(1-x)^{n-0} \\
& +f\left(\frac{1}{n}\right)\left(\begin{array}{l}
n \\
1
\end{array}\right) x(1 \\
& -x)^{n-1} \\
& +f\left(\frac{2}{n}\right)\left(\begin{array}{l}
n \\
2
\end{array}\right) x^{2}(1-x)^{n-2} \\
& +f\left(\frac{3}{n}\right)\left(\begin{array}{l}
n \\
3
\end{array}\right) x^{3}(1 \\
& +\cdots+f\left(\frac{n}{n}\right)\left(\begin{array}{l}
n \\
n
\end{array}\right) x^{n}(1-x)^{n-n} \\
& =f(0)(1-x)^{n} \\
& +f\left(\frac{1}{n}\right)\left(\frac{n !}{1 !(n-1) !}\right) x(1-x)^{n-1}+ \\
& f\left(\frac{2}{n}\right)\left(\frac{n !}{2 !(n-2) !}\right) x^{2}(1-x)^{n-2}+ \\
& f\left(\frac{3}{n}\right)\left(\frac{n !}{3 !(n-3) !}\right) x^{3}(1-x)^{n-3}+\cdots \\
& =f(0)(1-x)^{n}+f(1) x^{n} \\
& +n f\left(\frac{1}{n}\right) x(1-x)^{n-1} \\
& +\frac{n(n-1)}{2 !} f\left(\frac{2}{n}\right) x^{2}(1 \\
& -x)^{n-2}+
\end{aligned}
$$

$\frac{n(n-1)(n-2)}{3 !} f\left(\frac{3}{n}\right) x^{3}(1$

$$
-x)^{n-3}+\cdots+f(1) x^{n}
$$

By substituting $n=3$. Then

$$
\begin{aligned}
P(x)= & f(0)(1-x)^{3} \\
& +3 f\left(\frac{1}{3}\right) x(1-x)^{2} \\
& +3 f\left(\frac{2}{3}\right) x^{2}(1-x) \\
& +3 f\left(\frac{3}{3}\right) x^{3}(1-x)^{0}
\end{aligned}
$$

Let

$$
\begin{gathered}
f(0)=y_{0}, f\left(\frac{1}{3}\right)=y_{1}, f\left(\frac{2}{3}\right) \\
=y_{2}, f(1)=y_{3} \\
P(x)=y_{0}(1-x)^{3}+3 y_{1} x(1-x)^{2} \\
+3 y_{2} x^{2}(1-x) \\
+y_{3} x^{3}
\end{gathered}
$$

By integrating both sides of equation

(3) From 0 to 1, one can have:-

$$
\begin{gathered}
\int_{0}^{1} f(x) d x \simeq \int_{0}^{1} P(x) d x \\
=\int_{0}^{1}\left[y_{0}(1-x)^{3}+3 y_{1} x(1-x)^{2}\right. \\
+3 y_{2} x^{2}(1-x) \\
\left.+y_{3} x^{3}\right] d x
\end{gathered}
$$




$$
\begin{aligned}
=\frac{1}{4}\left(y_{0}+y_{1}+y_{2}+y_{3}\right) \\
=\frac{1}{4}\left(f_{0}+f_{1}+f_{2}+f_{3}\right)
\end{aligned}
$$

Now, by using the transformation

$x=a+t(b-a), h=\frac{b-a}{3}$ and above

equation, we get:-

$$
\begin{aligned}
\int_{a}^{b} f(x) d x= & \frac{3 h}{4}\left[f_{0}+f_{1}+f_{2}\right. \\
& \left.+f_{3}\right] \ldots(4)
\end{aligned}
$$

This formula is said to be modified Simpson's $3 / 8$ rule.

\section{The Composite Modified} Simpson's 3/8 Rule:

The Composite modified Simpson's $3 / 8$ rule can be derived by extending the modified Simpson's 3/8 rule.

This procedure is begin by dividing [a,b] into $\mathrm{n}$ subintervals ( $\mathrm{n}$ is multiple of three), and applying the modified Simpson's $3 / 8$ rule over each interval, then the sum of the results obtained for each interval isthe approximate value of integral, that is

$$
\begin{aligned}
& \int_{b}^{a} f(x) d x \\
& =\int_{a}^{a+3 h} f(x) d x+\int_{a+3 h}^{a+6 h} f(x) d x \\
& +\ldots \ldots+\int_{a+(n-6) h}^{a+(n-3) h} f(x) d x \\
& +\int_{a+(n-3) h}^{b} f(x) d x \quad, \text { where } h \\
& =\frac{b-a}{n} \\
& \int_{a}^{b} f(x) d x=\frac{3 h}{4}[f(a)+f(a+h) \\
& +f(a+2 h)+f(a \\
& +3 h)]
\end{aligned}
$$

$$
\begin{aligned}
& +\frac{3 h}{4}[f(a+3 h)+f(a+4 h) \\
& +f(a+5 h) \\
& +f(a+6 h)]+\cdots \\
& +\frac{3 h}{4}[f(a+(n-6) h) \\
& +f(a+(n-5) h) \\
& +f(a+(n-4) h) \\
& +f(a+(n-3) h)] \\
& +\frac{3 h}{4}[f(a+(n-3) h) \\
& +f(a+(n-2) h) \\
& +f(a+(n-1) h) \\
& +f(b)] \\
& =\frac{3 h}{4}[f(a)+f(a+h)+f(a+2 h) \\
& +2 f(a+3 h) \\
& +f(a+4 h) \\
& +f(a+5 h)+\cdots \\
& +2 f(a+(n-3) h) \\
& +f(a+(n-2) h) \\
& +f(a+(n-1) h) \\
& +f(b)] \\
& =\frac{3 h}{4}\left[f(a)+\sum_{j=1,4,7, \ldots}^{n-2}\left[f\left(x_{j}\right)\right.\right. \\
& \left.+f\left(x_{j+1}\right)\right] \\
& +2 \sum_{j=3,6,9 \ldots}^{n-3} f\left(x_{j}\right) \\
& +f(b) \quad] \ldots(5)
\end{aligned}
$$

This formula is said to be the composite modified Simpson's 3/8 rule.

\section{Numerical Solution for Solving The One-dimensional Volterra Linear Integral Equation Using The Composite Modified Simpson's 3/8 Rule:}

In this section, we use the composite modified Simpson's $3 / 8$ rule for solving the one-dimensionalVolterra linear integral equations of the second kind given by 


$$
\begin{gathered}
u(x)=f(x)+\lambda \int_{a}^{x} K(x, y) u(y) d y, \\
x \geq a \ldots(6)
\end{gathered}
$$

First, we divide the interval $[\mathrm{a}, \mathrm{b}]$ into $\mathrm{n} \quad$ subintervals $\left[x_{i}, x_{i+1}\right], i=$ $0,1,2, \ldots, n-1$, such that $x_{i}=a+$ $i h, i=0,1, \ldots, n$ where $n$ is multiple of three and $h=\frac{b-a}{h}$. So, the problem here is to find the numerical solution of equation (6) at each $x_{i}, i=0,1, \ldots, n$. Then by setting $x=x_{i}$ in equation (6), we get

$$
\begin{aligned}
& u\left(x_{i}\right) \\
& =f\left(x_{i}\right) \\
& +\lambda \int_{a}^{x_{i}} k\left(x_{i}, y\right) u(y) d y, \quad i \\
& =0,1, \ldots, n \ldots(7)
\end{aligned}
$$

For $i=3,6,9, \ldots n$.We approximate the integral that appeared in the right hand side of equation (7) by the composite modified Simpson's 3/8 rule to obtain:-

$$
\begin{aligned}
\begin{array}{l}
u_{0}=f_{0} \\
u_{i}=\frac{3 h}{4}\left[k\left(x_{i}, x_{0}\right) u_{0}\right.
\end{array} & +\sum_{j=1,4,7, \ldots}^{i-2}\left[k\left(x_{i}, x_{j}\right) u_{j}\right. \\
& \left.+k\left(x_{i}, x_{j+1}\right) u_{j+1}\right] \\
\left.+2 \sum_{j=3,6,9, . .} k\left(x_{i}, x_{j}\right) u_{j}+k\left(x_{i}, x_{i}\right) u_{i}\right] & i=3,6,9, \ldots, n \ldots(8)
\end{aligned}
$$

And, for $i \neq 3,6,9, \ldots, n$, we approximate the integral that appeared in the right hand side of equation (7) by the composite modified Trapezoidal rule [13] to get

$$
\begin{aligned}
u_{i}=f_{i}+\frac{\lambda h}{2}\left[k\left(x_{i}, x_{0}\right) u_{0}\right. \\
+2 \sum_{j=1}^{i-2}\left[k\left(x_{i}, x_{j}\right) u_{j}\right. \\
\left.\left.+k\left(x_{i}, x_{j+1}\right) u_{j}\right]\right], i \\
\quad \neq 3,6,9, \ldots, n . \ldots(9)
\end{aligned}
$$

To illustrate this method, we consider the following examples:

\section{Example (1):}

Consider the one-dimensional Volterra linear integral equation of the second kind:-

$$
u(x)=x+\frac{2}{5} \int_{0}^{x} x y u(y) d y \quad 0 \leq x
$$$$
\leq 2
$$

whose exact solution is $u(x)=x e^{\frac{x^{2}}{5}}$, this equation can be solved numerically with the composite modified Simpson's 3/8 rule. First, we divide the interval $[0,2]$ into 9 subintervals, such that

$x_{i}=\frac{2 i}{9}, \quad i=0,1, \ldots 9 . \quad$ Then $u_{0}=$ $f(0)=0$, and the equation becomes:-

$$
\begin{aligned}
& u_{i}=x_{i}+\frac{1}{30} \sum_{j=1,4,7, \ldots}^{i-2}\left(x_{i} x_{j} u_{j}\right. \\
& \left.+x_{i} x_{j} u_{j+1}\right) \\
& +\frac{1}{15} \sum_{j=3,6,9, \ldots}^{i-3} x_{i} x_{j} u_{j} \\
& +\frac{1}{30} x_{i}^{2} u_{i} \text {, } \\
& i=3,6 \text {, } \text {, }
\end{aligned}
$$

and the equation (9) becomes:-

$$
\begin{aligned}
u_{i}=x_{i}+\frac{2}{45} \sum_{j=1}^{i-1} x_{i} x_{j} u_{j} \\
\quad+\frac{1}{45} x_{i}^{2} u_{i}, \quad i \\
\quad \neq 3,6,9, \quad \ldots .
\end{aligned}
$$

By setting $i=1$ in the equation (11) one can get $u_{1}=0.2224663554$

By setting $i=2$ in the equation (11) one can get $u_{2}=0.4473848062$ By setting $i=3$ in the equation (10) one can get $u_{3}=0.6822919096$.

By continuing in this manner one can get the following values: 


$\begin{array}{lll}\mathrm{U}_{0}=0 & \mathrm{U}_{1}=0.2224663554 & \mathrm{U}_{2}=0.4473848062 \\ \mathrm{U}_{3}=0.6822919096 & \mathrm{U}_{4}=0.9330498672 & \mathrm{U}_{5}=1.2202686732 \\ \mathrm{U}_{6}=1.5752706589 & \mathrm{U}_{7}=2.0084545071 & \mathrm{U}_{8}=2.6015170971\end{array}$

Second, we divide the interval $[0,2]$ into 18 subintervals, such that

$x_{i}=\frac{i}{9}, \quad i=0,1, \ldots, 18 . \quad$ Then $u_{0}=$ $f(0)=0$, and the equations (8), (9) become:-

$$
\begin{aligned}
u_{i}=x_{i}+\frac{1}{60}[ & \sum_{j=1,4,7,--}^{i-2}\left(x_{i} x_{j} u_{j}\right. \\
& \left.+x_{i} x_{j+1} u_{j+1}\right) \\
& +2 \sum_{j=3,6,9,--}^{i-3} x_{i} x_{j} u_{j} \\
& \left.+x_{i}^{2} u_{i}\right]
\end{aligned}
$$

$$
\begin{aligned}
& \mathrm{U}_{0}=0 \\
& \mathrm{U}_{3}=0.3342955701 \\
& \mathrm{U}_{6}=0.6805480476 \\
& \mathrm{U}_{9}=1.0704371891 \\
& \mathrm{U}_{12}=1.5650052777 \\
& \mathrm{U}_{15}=2.2770419403 \\
& \mathrm{U}_{18}=3.4276679769
\end{aligned}
$$

$$
\begin{aligned}
& \mathrm{U}_{1}=0.1111263548 \\
& \mathrm{U}_{4}=0.4471364570 \\
& \mathrm{U}_{7}=0.8028413818 \\
& \mathrm{U}_{10}=1.2182194913 \\
& \mathrm{U}_{13}=1.7675583096 \\
& \mathrm{U}_{16}=2.5897590702
\end{aligned}
$$

Third, we divide the interval $[0,2]$ into 36 and 72 subintervals such that

$$
\begin{aligned}
x_{i}=\frac{i}{18}, \quad i & =0,1,2, \ldots, 36, \quad x_{i} \\
& =\frac{i}{36}, i \\
& =0,1,2, \ldots, 72
\end{aligned}
$$

$$
\begin{aligned}
& \mathrm{U}_{2}=0.2224052300 \\
& \mathrm{U}_{5}=0.5620748374 \\
& \mathrm{U}_{8}=0.9318813651 \\
& \mathrm{U}_{11}=1.3813511291 \\
& \mathrm{U}_{14}=2.0014643364 \\
& \mathrm{U}_{17}=2.9658216063
\end{aligned}
$$

Table (1) represents the exact and the numerical solution of example (1)at specific points for different values of $n$

\begin{tabular}{|c|l|l|c|c|c|}
\hline \multirow{2}{*}{$\mathbf{X}$} & \multirow{2}{*}{ Exact Solution } & \multicolumn{4}{|c|}{ Numerical Solution } \\
\cline { 3 - 6 } & & $\mathbf{N}=\mathbf{9}$ & $\mathbf{N}=\mathbf{1 8}$ & $\mathbf{N}=\mathbf{3 6}$ & $\mathbf{N}=\mathbf{7 2}$ \\
\hline 0.222222222 & 0.2223848585 & 0.2224663554 & 0.2224052300 & 0.2223899536 & 0.2223861320 \\
\hline 0.444444444 & 0.4470533010 & 0.4473848062 & 0.4471364570 & 0.4470740553 & 0.4470584955 \\
\hline 0.6666666667 & 0.6799663130 & 0.6822919096 & 0.6805480476 & 0.6801117669 & 0.6800026778 \\
\hline 0.888888889 & 0.9314983085 & 0.9330498672 & 0.9318813651 & 0.9315949132 & 0.9315223638 \\
\hline 1.111111111 & 1.2175126789 & 1.2202686732 & 1.2182194913 & 1.2176873746 & 1.2175566340 \\
\hline 1.333333333 & 1.5615934837 & 1.5752706589 & 1.5650052777 & 1.5624459538 & 1.5618065712 \\
\hline 1.555555556 & 1.9992459998 & 2.0084545071 & 2.0014643364 & 1.9998129746 & 1.9993862624 \\
\hline 1.777777778 & 2.5855576010 & 2.6015170971 & 2.5897590702 & 2.5865822277 & 2.5858171455 \\
\hline
\end{tabular}




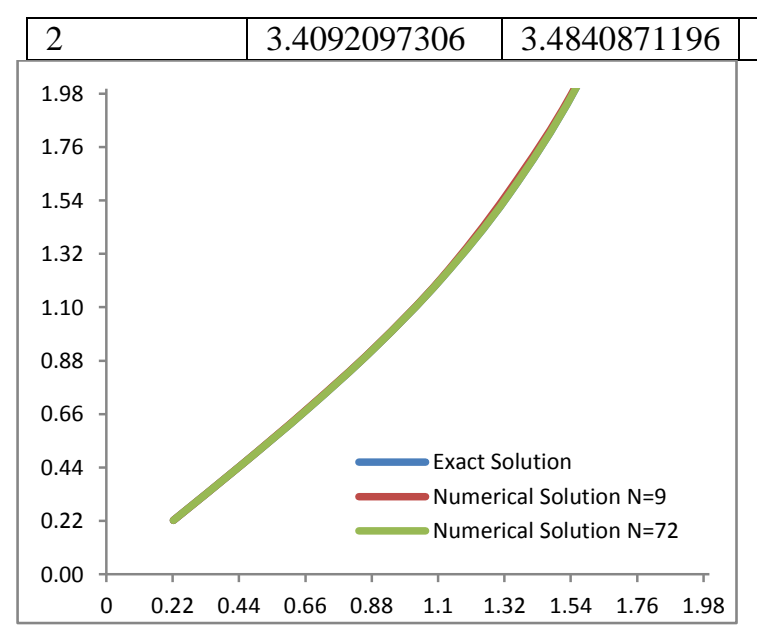

Fig(1): Graph of exact and numerical solution of example 1 .

\section{Example(2):}

Consider the one-dimensional Volterra linear integral equation of the second kind:-

$$
\begin{aligned}
u(x)=x-\frac{4}{35} & x^{\frac{7}{2}} \\
& +\int_{0}^{x}(x \\
& -y)^{\frac{3}{2}} u(y) d y \quad 0 \leq x \\
& \leq 2
\end{aligned}
$$

Whose exact solution is $u(x)=x$. We solve this equation numerically with the composite modified Simpson's 3/8 rule. First, we divide the interval $[0,2]$ into 9 subintervals such that \begin{tabular}{|l|l|l|}
3.4276679769 & 3.4134233850 & 3.4103583731 \\
\hline
\end{tabular} $x_{i}=\frac{2 i}{9}, \quad i=0,1, \ldots, 9 . \quad$ Then $u_{0}=$ $f(0)=0$, and the equations (8),(9) become:-

$$
\begin{aligned}
u_{i}=x_{i}-\frac{4}{35} x_{i}^{\frac{7}{2}} & \\
& +\frac{1}{6}\left\{\sum _ { j = 1 , 4 , 7 , . . } ^ { i - 2 } \left[\left(x_{i}\right.\right.\right. \\
& \left.-x_{j}\right)^{\frac{3}{2}} u_{j}+\left(x_{i}\right. \\
& \left.\left.\left.-x_{j+1}\right)^{\frac{3}{2}} u_{j+1}\right]\right\}
\end{aligned}
$$

$+\frac{1}{3} \sum_{j=3,6,9, . .}^{i-3}\left(x_{i}-x_{j}\right)^{\frac{3}{2}} u_{j}, \quad i$

$$
=3,6,9, \quad \ldots(14)
$$

and

$$
\begin{array}{r}
u_{i}=x_{i}-\frac{4}{35} x_{i}^{\frac{7}{2}}+\frac{2}{9} \sum_{j=1}^{i-1}\left(x_{i}-x_{j}\right)^{\frac{3}{2}} u_{i}, \\
i \neq 3,6,9, \quad \ldots .(15)
\end{array}
$$

By setting $i=1$ in equation (15) one can get $u_{1}=0.2216310035$.

By setting $i=2$ in equation (15) one can get $u_{2}=0.4429149690$.

By setting $i=3$ in equation (14) one can get $u_{3}=0.6576958875$.

And, by continuing in this manner one can get the following values:-
$\mathrm{U}_{0}=0$
$\mathrm{U}_{1}=0.2216310035$
$\mathrm{U}_{2}=0.4429149690$
$\mathrm{U}_{3}=0.6576958875$
$\mathrm{U}_{4}=0.8844957113$
$\mathrm{U}_{5}=1.1046072143$
$\mathrm{U}_{6}=1.3079521615$
$\mathrm{U}_{7}=1.5427891825$
$\mathrm{U}_{8}=1.7602438383$

$\mathrm{U}_{9}=1.9464920052$

Second, if we divide the interval $[0,2]$ into 18 subintervals, such that

$$
\begin{aligned}
u_{i}=x_{i}-\frac{4}{35} x_{i}^{\frac{7}{2}} & \\
& +\frac{1}{12} \sum_{j=1,4,7,--}^{i-2}\left[\left(x_{i}\right.\right. \\
& \left.-x_{j}\right)^{\frac{3}{2}} u_{j} \\
& \left.+\left(x_{i}-x_{j+1}\right)^{\frac{3}{2}} u_{j+1}\right]
\end{aligned}
$$$$
x_{i}=\frac{i}{9}, \quad i=0,1, \ldots, 18 \text {. Then the }
$$$$
\text { equations (8), (9) become:- }
$$ 


$$
\begin{aligned}
& +\frac{1}{6} \sum_{j=3,6,9, \ldots}^{i-3}\left(x_{i}-x_{j}\right)^{\frac{3}{2}} u_{j}, \quad i \\
& =3,6,9, \ldots, 18 . \quad \ldots(16)
\end{aligned}
$$

and

$$
\begin{aligned}
u_{i}=x_{i}-\frac{4}{35} x_{i}^{\frac{7}{2}} & \\
& +\frac{1}{9} \sum_{j=1}^{i-1}\left(x_{i}\right. \\
& \left.-x_{j}\right)^{\frac{3}{2}} u_{j}, i \\
& \neq 3,6,9, \ldots, 18 .
\end{aligned}
$$$$
\mathrm{u}_{0}=0
$$$$
\mathrm{u}_{3}=0.3325444915
$$$$
\mathrm{u}_{6}=0.6645903134
$$$$
\mathrm{u}_{9}=0.9962027164
$$$$
\mathrm{u}_{12}=1.3273025035
$$$$
\mathrm{u}_{15}=1.6577165689
$$$$
\mathrm{u}_{18}=1.9871689996
$$

$$
\begin{aligned}
& \mathrm{u}_{1}=0.1110588543 \\
& \mathrm{u}_{4}=0.4440838939 \\
& \mathrm{u}_{7}=0.7769297456 \\
& \mathrm{u}_{10}=1.1095483963 \\
& \mathrm{u}_{13}=1.4418215959 \\
& \mathrm{u}_{16}=1.7735580777
\end{aligned}
$$

By setting $i=1$ in equation (17) one can get $u_{1}=0.1110588543$.

By setting $i=1$ in equation (17) one

\begin{tabular}{|c|c|c|c|c|c|}
\hline \multirow{2}{*}{$\mathbf{X}$} & \multirow{2}{*}{ Exact Solution } & \multicolumn{4}{|c|}{ Numerical Solution } \\
\hline & & $N=9$ & $N=18$ & $N=36$ & $\mathrm{~N}=72$ \\
\hline 0.222222222 & 0.2222222222 & 0.2216310035 & 0.2220880359 & 0.2221907850 & 0.2222147077 \\
\hline 0.444444444 & 0.4444444444 & 0.4429149690 & 0.4440838939 & 0.4443581710 & 0.4444235479 \\
\hline 0.6666666667 & 0.6666666667 & 0.6576958875 & 0.6645903134 & 0.6661767275 & 0.6665490295 \\
\hline 0.888888889 & 0.8888888889 & 0.8844957113 & 0.8878314093 & 0.8886332252 & 0.8888264788 \\
\hline 1.111111111 & 1.1111111111 & 1.1046072143 & 1.1095483963 & 1.1107322356 & 1.1110185287 \\
\hline 1.333333333 & 1.3333333333 & 1.3079521615 & 1.3273025035 & 1.3318853591 & 1.3329816693 \\
\hline 1.555555556 & 1.5555555556 & 1.5427891825 & 1.5524695371 & 1.5548073905 & 1.5553725636 \\
\hline 1.777777778 & 1.7777777778 & 1.7602438383 & 1.7735580777 & 1.7767539344 & 1.7775274066 \\
\hline 2 & 2.0000000000 & 1.9464920052 & 1.9871689996 & 1.9969012387 & 1.9992444590 \\
\hline
\end{tabular}
can get $u_{2}=0.4429149690$.

By setting $i=3$ in equation (16) one can get $u_{3}=0.3325444915$

And, by continuing in this manner one can get the following values:-
Third, we divide the interval $[0,2]$ into 36 and 72 subintervals such that

$$
\begin{aligned}
x_{i}=\frac{i}{18}, \quad i & =0,1, \ldots, 36 \text { and } x_{i} \\
& =\frac{i}{36}, \quad i=0,1, \ldots, 72
\end{aligned}
$$

$$
\begin{aligned}
& \mathrm{u}_{2}=0.2220880359 \\
& \mathrm{u}_{5}=0.5550527639 \\
& \mathrm{u}_{8}=0.8878314093 \\
& \mathrm{u}_{11}=1.2203506870 \\
& \mathrm{u}_{14}=1.5524695371 \\
& \mathrm{u}_{17}=1.8839681446
\end{aligned}
$$

Table (2)represents the exact and the numerical solution of example (2) at specific points for different values of $n$ 


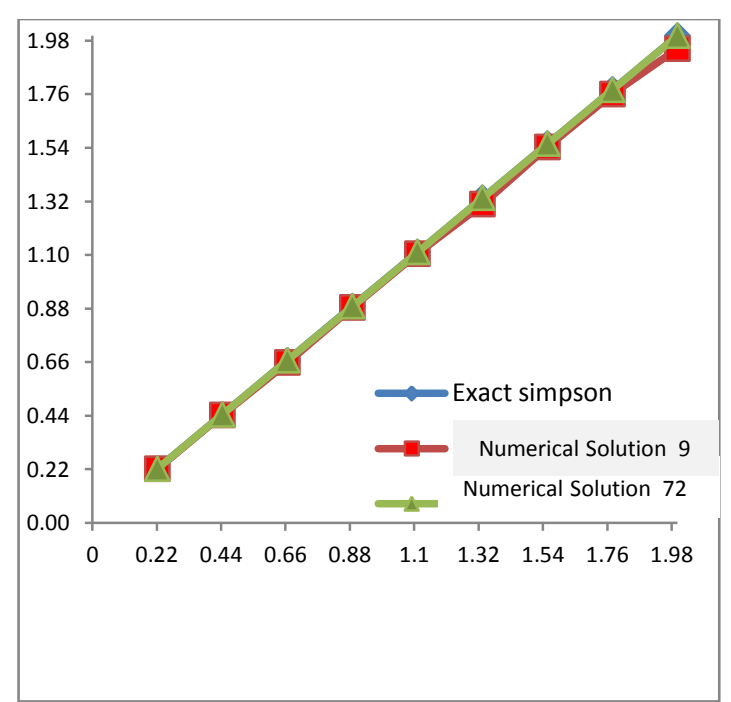

Fig (2): Graph of exact and numerical solution of example 2

\section{References:}

1. Rahmann, M. 2007. Integral Equations and their Applications, WIT Press, Boston.

2. Hochstadt, H. 1973. Integral Equations. AWiley-Interscience publication,New York.

3. Terry, A.H. 1985. Introduction to Integral Equations with Applications. Marcel Dekker INC., New York.

4. Al-Rawi, S. 1995. Numerical solution of the first kind Integral equations of convolution Type. M.Sc. Thesis, University of Technology, Baghdad.

5. Al.Nasir, R. 2000. Nemerical Solution of Volterra Integral Equations of the second kind. M.Sc Thesis, University of Technology.
6. Deleves, L. and Mohammed, J. 1985. Computational Methods of Integral Equations. Cambridge University press.

7. Davies, A. 1980.The finite element method. A First Approach published in the united states of Oxford University press, New York.

8. Stoer, J. and Bulirsch, R. 1993. Introduction to Numerical Analysis of the second kind. SpringerVerlage, Berlin.

9. Stark, A.P. 1970. Introduction to Numerical Methods. MacmillanPublishing Co. Inc., New York.

10. Jafar, S. and Mahdi, H. 2007. A generalized block-by-block method forSolving linear Volterra integral equations. Applied Mathematicsand Computation. 188(2): 1969-1974.

11. Mladen, M. and Eva, O. 2007. An application of Romberg extrapolation on quadrature method for solving linear Volterra integral equations of the second kind. Applied Mathematics and Computation. 194(2):389-393.

12.Shaima, M. 2008. Some Modified Quadrature For Solving Systems of Volterra Linear Integral Equations. M.Sc.Thesis, College of Education, Ibn- AL-Haitham, University of Baghdad .

13.Kenneth, I.Toy. 2000. Bernstein Polynomials on line geometrical modeling notes. University of California, USA.

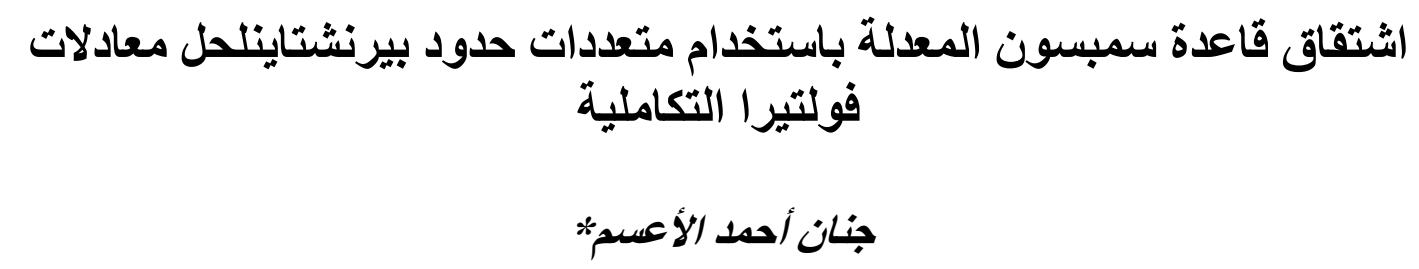

*قسم الرياضيات ـ كلية العلوم للبنات- جامعة بغداد ـ العراق 


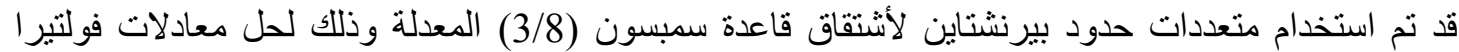
التكامليةالخطية منالنوع الثاني.وتبين ان الحل باستخدام هذه الطريقة قريب جدا من الحل التحليلي (المضبوط). 